\title{
A New Vesicle-loaded Hydrogel System Suitable for Topical Applications: Preparation and Characterization
}

\author{
Carlotta Marianecci ${ }^{\mathrm{a}}$, Maria Carafa ${ }^{\mathrm{a}}$, Luisa Di Marzio ${ }^{\mathrm{b}}$, Federica Rinaldi ${ }^{\mathrm{a}}$, Chiara Di Meo ${ }^{\mathrm{a}}$, Franco Alhaique ${ }^{\mathrm{a}}$, Pietro \\ Matricardi ${ }^{\mathrm{a}}$, Tommasina Coviello ${ }^{\mathrm{a}}$ \\ a Department of Drug Chemistry and Technologies, "Sapienza”, University of Rome, Rome Italy \\ b Department of Drug Science, University of Chieti “G. D’Annunzio”, Chieti, Italy
}

Received, July 18, 2011; Revised, July 26, 2011; Accepted, September 5, 2011; Published, September 5, 2011.

\begin{abstract}
Purpose. Aim of this research was to prepare and study drug release from a new formulation consisting of non ionic surfactant vesicular structures, niosomes (NSVs), loaded with model molecules calcein (CALC), nile red (NR), ibuprofen (IBU) or caffeine (CAFF), and embedded in a hydrogel matrix. Methods. The system locust bean gum/xanthan $(1: 1)$, prepared at $60{ }^{\circ} \mathrm{C}$, was used to entrap the vesicles (Tween 20/cholesterol 1:1), loaded with guest molecules and the release profiles were detected at 32 ${ }^{\circ} \mathrm{C}$. The hydrogel systems were characterized by means of scanning electron microscopy; niosomes were characterized by means of size and $\zeta$-potential measurements. Results. Size measurements showed that a slight increase in vesicle dimensions occurs after inclusion of CALC or CAFF (hydrophilic molecules) in the vesicular structures. $\zeta$-potential measurements showed that the inclusion of these molecules did not significantly modify the surface charge of empty vesicles. This was probably related to an almost negligible drug adsorption on the vesicle surface. The release from the niosomes-gel systems of two probes (CALC and NR) showed that the diffusion of CALC through the gel was not affected by the niosome entrapment while for NR, the presence of vesicles was crucial. The release profiles from niosomes-gel systems and from the hydrogel alone of model drugs, CAFF and IBU, showed an appreciable difference between the two drugs: the more hydrophilic CAFF was released much faster than IBU. In all release studies turbidity, dimension and $\zeta$-potential analyses indicated that the loaded niosomes were released by the hydrogel matrix without being damaged. Conclusions. The reported in vitro experiments show the capability of the novel formulation to combine the qualities of both chosen single systems, i.e. the niosomes and the polymeric network. The hydrogel shows a protective effect on vesicle integrity and leads to a slow release of the loaded model molecules from the polysaccharidic system.
\end{abstract}

This article is open to POST-PUBLICATION REVIEW. Registered readers (see "For Readers") may comment by clicking on ABSTRACT on the issue's contents page.

\section{INTRODUCTION}

The major disadvantage of transdermal and dermal drug delivery is the poor penetration of most compounds across human skin. The main barrier of the skin is located within its uppermost layer, the stratum corneum. Several approaches have been developed to weaken this skin barrier. One of the approaches to achieve increased skin penetration of drugs and other cosmetic chemicals, is through the use of vesicular systems, such as liposomes and niosomes. These carriers can act as drug reservoirs showing several advantages over conventional dosage forms. Furthermore, by modifying their composition or their surface it is possible to adjust drug release rate and/or the affinity for the target site. Colloidal systems, like micro-spheres, appeared in the fifties, and they were mainly used in the field of cosmetics. Liposomes, discovered in the sixties, appeared in the cosmetic market in 1986 (1), and for a long time, they were considered as the main innovative contributors in the dermal area for both pharmaceutical and cosmetic products. Due to some drawbacks like high cost, variable purity of natural phospholipids and unstable nature, surfactant based vesicles 'niosomes' were proposed. While liposomes are unilamellar or multilamellar structures composed of lipid molecules (most often phospholipids) (1), niosomes are formed by non-ionic surfactants that in aqueous media assume closed bilayer structures (2). In comparison to phospholipid vesicles (liposomes), niosomes offer higher chemical stability, lower costs, and great availability of surfactants with different structures (3-5).

Corresponding Author: Maria Carafa; Department of Drug Chemistry and Technologies; "Sapienza", University of Rome, Rome, Italy; E-mail:maria.carafa@uniroma1.it 
These vesicular delivery systems attracted considerable attention in topical/transdermal drug delivery for many reasons: niosomes can act as enhancers for the penetration through the skin of guest molecules, are biodegradable, effective in the modulation of drug release properties, and in most cases non-toxic. Their effectiveness is strongly dependent on their physico-chemical properties, such as composition, size, charge and lamellarity (6). Niosomes, as liposomes, can carry hydrophilic and/or hydrophobic drugs. In 1975 the first cosmetic product containing non-ionic surfactant vesicles, called 'Niosomes', was brought into the market (7-8). Later the product had also a successor, the 'Niosome Plus' antiageing cream, which reached the market in the early nineties. Usually, vesicles are applied to the skin in solution or in gels, since stable niosomal creams are difficult to formulate and gel formulations do not seem to prevent efficient transport of niosomes into the skin (9).

Aim of this research was the study of the technological properties of a new formulation consisting of model drugs loaded within vesicular structures embedded in a hydrogel matrix. In particular, the system was tailored in order to obtain a protection of the vesicle integrity within the hydrogel and to achieve a slow diffusion of such vesicles inside the gel network.

Ibuprofen (IBU) (10) and caffeine (CAFF) (11) were chosen as model drugs for topical application, according to their distribution coefficient (IBU: $\log \mathrm{D}=1.21$; CAFF: $\log \mathrm{D}=$ 0.10 ) and pKa (IBU: $\mathrm{pKa}=4.3$; CAFF: $\mathrm{pKa}=$ $0.51)$.

For the choice of the polymeric matrix (12-13) to be used as a carrier for the loaded vesicles, different systems were tested and the gel prepared with locust bean gum (LBG) and xanthan (xanth) (Figure 1) was finally selected. These two polysaccharides exhibit, as it happens for some other binary mixtures, unexpected synergistic interactions. In fact, the mixture of the two polymer solutions may form a gel under conditions where the individual components are non-gelling, i.e. a synergistic gelation occurs by a direct binding between the two polymers (14).
Locust Bean Gum

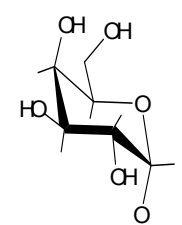

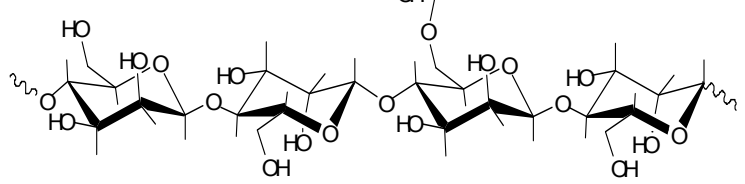

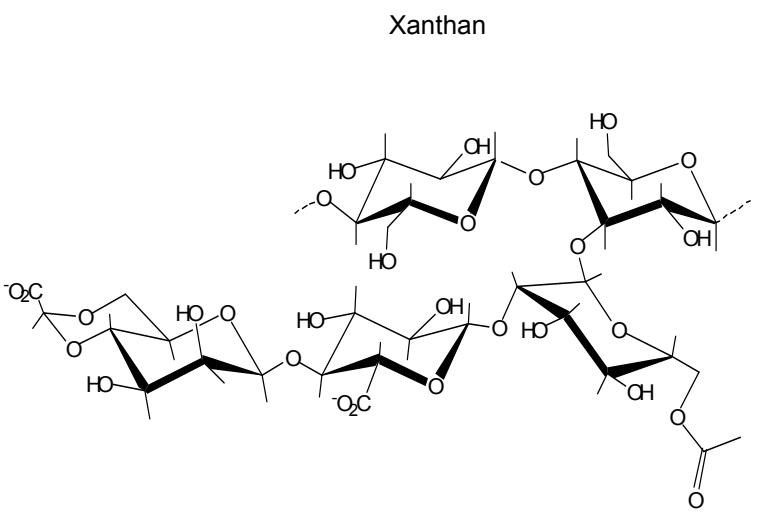

Polysorbate 20 (Tween $\left.{ }^{\circledR} 20\right)$

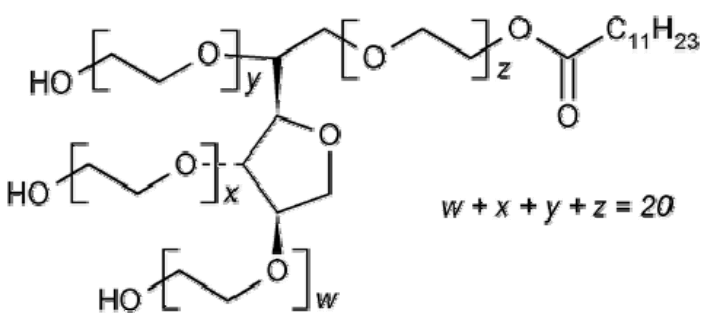

Figure 1. Repeating units of locust bean gum,xanthan, and Tween 20. 
Furthermore, the strength of the network depends on the temperature during preparation and on the weight ratio between the two components. Thus, the mechanical properties of the gel can be modulated by varying the relative amount of the two polymers and taking also into account the different conformation of xanth at $25^{\circ}$ C (double-helix ordered structure) (15). The strongest network is obtained when the two polymers are present in a weight ratio $1: 1$ and the two solutions are mixed at temperature higher than $45^{\circ} \mathrm{C}$, i.e. when xanth is dissolved as single chains. However, a wide debate is still open in elucidating the mechanism leading to the gel formation and several models were proposed (1721).

One important aspect related to the use of this binary mixture is supported also by the fact that these polymers are biocompatible and they are already widely used in food industry (22-27). Furthermore, it must be pointed out that the single polymers are already used as excipients in tablet formulations, and the LBG/xanthan gels have been proposed in pharmaceutical applications for modified release purposes (28-35).

In the present work the system LBG/xanth (1:1), prepared at $60^{\circ} \mathrm{C}$, was used to entrap the vesicles previously loaded with guest molecules and the release profiles have been detected at $32^{\circ}$ $\mathrm{C}$, considered as surface skin temperature. The compatibility of the vesicles with the hydrogel network was studied and the advantages of the presence of both components, gel and vesicles, are discussed. The gel slows down the release of niosomes, and the vesicles should enhance drug absorption.

\section{MATERIALS AND METHODS}

\section{Materials}

Locust Bean Gum (LBG), from Ceratonia Campestris, was provided by Carbomer (San Diego, USA). The ratio between Mannose and Galactose, was estimated by means of ${ }^{1} \mathrm{H}$ NMR (carried out at $70^{\circ} \mathrm{C}$ with a Brucker AVANCE AQS 600 spectrometer, operating at $600.13 \mathrm{MHz}$ ) and an $\mathrm{M} / \mathrm{G}$ value of $\sim 3.4$ was found. The average molecular weight $\left(\mathrm{M}_{\mathrm{w}}\right)$ of LBG, 5.0x $10^{5}$ $(\mathrm{g} / \mathrm{mol})$, was estimated from static light scattering measurements (36).

Xanthan Gum, from Xanthomonas campestris, was provided by Fluka (Milan, Italy). For each repeating unit, 1.6 acetate and 2.7 pyruvate groups were estimated by ${ }^{1} \mathrm{H}$ NMR measurements (carried out at $85^{\circ} \mathrm{C}$ with a Brucker AVANCE AQS 600 spectrometer, operating at 600.13
$\mathrm{MHz}$ ). The average molecular weight of xanth, $1.25 \times 10^{6}(\mathrm{~g} / \mathrm{mol})$, was obtained by means of static light scattering measurements (36).

The two polysaccharides were used after purification. A given amount of xanth, sodium salt, (polymer concentration, $\mathrm{c}_{\mathrm{p}}=0.5 \% \mathrm{w} / \mathrm{V}$ ) was dissolved in distilled water, under magnetic and mechanical stirring, at room temperature for $48 \mathrm{~h}$, while LBG $\left(c_{p}=0.5 \% \mathrm{w} / \mathrm{V}\right)$ was dispersed in distilled water, under magnetic and mechanical stirring, at $80^{\circ} \mathrm{C}$ for $24 \mathrm{~h}$ and at room temperature for 24 additional hours (37). The solutions were then exhaustively dialyzed at $4^{\circ} \mathrm{C}$ against distilled water, using dialysis membranes with a cut-off $12,000-14,000$. In order to convert the xanth polymer into the sodium form, $\mathrm{NaOH} 0.2 \mathrm{~N}$ was added to the dialyzed solution up to $\mathrm{pH}=7.0$. Finally, the samples were freeze-dried and stored in a desiccator until use. Tween 20 and Sephadex G-75, Hepes salt $\{\mathrm{N}-(2$-hydroxyethyl) piperazine$\mathrm{N}$-(2- ethanesulfonicacid)\}, cholesterol (CHOL), theophylline (TPH), caffeine (CAFF), ibuprofen (IBU) and calcein (CALC) were purchased from Sigma-Aldrich (Milan, Italy). 9-(diethylamino)5H-benzo[R] phenoxazin-5-one) (nile red, NR) was purchased from Acros Organics (Geel, Belgium). All other products and reagents were of analytical grade.

\section{Vesicle preparation and purification}

NSVs were prepared using Tw20 (Figure 1) and CHOL in 1:1 molar ratio $(15: 15 \mathrm{mM})$. Tw20 concentration in the samples was always remarkably above its CMC. Where indicated, the fluorescent probe NR $(2.5 \mathrm{mM})$ or IBU $(1 \%)$ or CAFF (3\%) were added to the surfactant/CHOL mixture before vesicle preparations. The vesicles were obtained by the "film" method, as previously reported (9). The dried films were hydrated by addition of HEPES buffer $(10 \mathrm{mM}, \mathrm{pH}=7.4)$ alone or sodium CALC $10^{-2} \mathrm{M}$ in HEPES $(10 \mathrm{mM}$, pH 7.4). The surfactant dispersion was mechanically stirred for about $5 \mathrm{~min}$ and then sonicated for 5 $\min$ at $60^{\circ} \mathrm{C}$ (Vibracells-VCX400-Sonics), equipped with an exponential microprobe operating at $23 \mathrm{kHz}$ and an amplitude of $6 \mathrm{~mm}$. Vesicle dispersions were purified by means of size exclusion chromatography on Sephadex G75 (mobile phase: HEPES buffer $10 \mathrm{mM}, \mathrm{pH}=7.4$ ).

\section{Vesicle characterization}

\section{Size, potential and morphology measurements}

The size and the $\zeta$-potential of the surfactant vesicle dispersions were measured by dynamic light scattering (DLS) with a Malvern Zetasizer Nano ZS90 (Malvern Instruments Ltd., 
Worcestershire, United Kingdom), which measures the mass distribution of particle size as well as the electrophoretic mobility of the dispersed particle.

The reported value represents the mean of the $\zeta$-potential $(\mathrm{mV})$ and the mean of the hydrodynamic diameter $(\mathrm{nm})$ of the surfactant vesicles. NSVs were diluted 1:100 and 1:10 in HEPES buffer for size and $\zeta$-potential measurements, respectively. It should be pointed out that size distribution results were determined as $\%$ of intensity of the colloidal dispersion. The polydispersity index (p.i.) value was determined as a measurement of the breadth of the size distribution: a p.i. value lower than 0.3 indicates a rather homogenous and monodisperse population (38). Size and $\zeta$-potential measurements were also performed to assess the delivery of intact vesicle in the surrounding medium during release experiments.

\section{Entrapment Efficiency (EE)}

Drug entrapment within the vesicles was assessed using a spectrofluorometer or a spectrophotometer, depending on molecule features, on purified vesicles, after their disruption with isopropanol (vesicle dispersion/isopropanol 1/1). Drug EE was calculated as follows:

$$
\begin{aligned}
\text { EE. }= & (\text { mass of incorporated drug/mass used for } \\
& \text { vesicle preparation }) \times 100
\end{aligned}
$$

\section{Preparation of the LBG/Xanthan hydrogel loaded with the vesicles.}

The LBG/Xanthan hydrogels were prepared by mixing appropriate amounts of LBG and xanth solutions $\left(\mathrm{c}_{\mathrm{p}}=0.5 \% \mathrm{w} / \mathrm{V}\right)$ previously autoclaved at $121^{\circ} \mathrm{C}$ for $20 \mathrm{~min}$. Aliquots $(4 \mathrm{~mL})$ of each polymer solution were mixed for $15 \mathrm{~min}$ at $60^{\circ} \mathrm{C}$ ( $\mathrm{T}>\mathrm{T}_{\text {melting }}$ of the Xanth chains) in the presence of $2 \mathrm{~cm}^{3}$ of the vesicle solutions in order to obtain a homogeneous system. The added vesicles were previously loaded with the different model molecules: CALC and CAFF as hydrophilic models and NR and IBU as hydrophobic ones. All prepared new formulations were still self sustaining gels.

\section{Release experiments}

The gels of LBG/Xanth loaded alternatively with the solution or suspensions of the model drugs and with the vesicles containing the model molecule were tested for the release experiments. Release experiments from the drug loaded-NSVs/gel system and from the drug/gel system (i.e. without the NSVs) were carried out in a beaker containing
$200 \mathrm{~cm}^{3}$ of HEPES. The set-up was kept at $\mathrm{T}=$ $32^{\circ} \mathrm{C}$ by means of a temperature-controlled water bath and the release medium was gently magnetically stirred during the experiment. Aliquots of $3 \mathrm{~mL}$ were withdrawn from the solution at appropriate time intervals and replaced with the same amount of fresh solvent. Released CALC was detected at Ex/Em 492/520 nm by means of a Perkin-Elmer LS50B spectrofluorometer (high pressure Xenon source) IBU, CAFF and NR were detected, respectively, at $272 \mathrm{~nm}, 273 \mathrm{~nm}$ and $574 \mathrm{~nm}$ by means of a spectrophotometer (Perkin-Elmer, lambda 3a, UV-Vis spectrometer) equipped by $1.0 \mathrm{~cm}$ pathlength quartz cells. All aliquots were analyzed immediately after sampling and addition of isopropanol $(1 / 1 \mathrm{v} / \mathrm{v})$ to disrupt the vesicular structures present in the medium. At the end of release experiments the presence of vesicular structures in the medium was assessed by turbidity measurements carried out at Ex/Em $600 / 600 \mathrm{~nm}$ by means of the Perkin Elmer spectrofluorometer and by size and $\zeta$-potential measurements.

All release experiments were carried out in triplicate. The values reported in the present paper represent the mean values and lay within $10 \%$ of the mean.

\section{Scanning electron microscopy (SEM)}

The SEM images were obtained using a FEI Quanta 400 FEG apparatus. Freeze-dried hydrogels were mounted on appropriate stubs and examined under vacuum $(50 \mathrm{~Pa})$, at an accelerating voltage of $15 \mathrm{KV}$. All images were acquired digitally at 400 and $800 x$ magnification.

\section{Statistics}

Results are expressed as the mean of three experiments \pm S.D. Statistical data analysis was performed using the Student's t-test at $p \leq 0.05$.

\section{RESULTS}

Vesicle characterization - NSVs
characterization Prepared formulations were compared to empty NSVs in terms of dimensions and zeta potential, to verify how the inclusion of model drugs can influence vesicular structure and properties.

Size measurements (Table 1) showed that a slight increase in vesicle dimensions occurs when CALC or CAFF are loaded in the vesicular structures. Zeta potential measurements (Table 1) showed that the inclusion of these molecules in 
NSVs did not significantly modify the surface charge of empty vesicles.

Loaded vesicles were also characterized. The EE was rather low, in comparison to other vesicular formulations (39-40).

Due to the low values of EE, release studies were carried out on non purified samples with guest molecules only partially loaded inside the vesicular structures (41-43).

The hydrogel effect on the structural integrity of vesicles during the release process, was also studied.

\section{Release experiments}

Release of hydrophilic and hydrophobic probes

The release from the NSVs-gel systems of two probes, the hydrophilic CALC and the hydrophobic NR, is shown in Figure 2, together with the release from the polysaccharidic gel matrix, i.e. without NSVs. The more hydrophilic CALC was completely released within the first 24 $h$ in both cases, when it was homogenously loaded inside the gel and when it was previously loaded inside the vesicles.

A completely different behaviour was detected in the case of the hydrophobic probe, NR. The results showed that the presence of vesicles loaded with NR was crucial.

\section{Release of hydrophilic and hydrophobic drugs.} The vesicles were also loaded with two model drugs, CAFF and IBU. The release profiles, obtained from the NSVs-gel systems and from the hydrogel without vesicles, are reported in Figure 3. The delivery of the two drugs were different: the more hydrophilic CAFF (solubility in water, $21.7 \mathrm{mg} / \mathrm{mL}, 25^{\circ} \mathrm{C}$ ) was released much faster than IBU and it was capable to diffuse out completely from the polymeric matrix within the first 24-48 hours, with a slight lower delivery rate in the presence of NSVs.
A rather different behaviour was detected when IBU, i.e. a hydrophobic drug (very slightly soluble in water, $<1 \mathrm{mg} / \mathrm{mL}, 25^{\circ} \mathrm{C}$ ), was tested. The results showed that, even after $48 \mathrm{~h}$, the release was not complete and only about $60 \%$ of the loaded drug was capable to diffuse from the matrix (Figure 3a). Also in this case there was only a slight difference between the two release profiles during the first 8 hours

\section{Scanning electron microscopy characterization} In Figure 4 the photographs of the two single polymers (LBG and xanth) and of the sample prepared at $60^{\circ} \mathrm{C}$ with a weight ratio of $1: 1$ are shown. It is interesting to appreciate the variations between the initial aspect of the single polymers, and the final situation, when the synergistic effect changed dramatically also the macroscopic morphology of the mixed system.

\section{DISCUSSION}

The reported formulations were analyzed in terms of dimensions and zeta potential, to verify how the inclusion of model drugs can influence vesicular structure and properties. Size measurements (Table 1) showed that CALC or CAFF (i.e. the more hydrophilic molecules) lead to a slight increase in vesicle dimensions. Zeta potential measurements (Table 1) showed that the inclusion of model molecules in NSVs did not modify significantly the surface charge of empty vesicles. These results were probably related to an almost negligible drug adsorption on the vesicle surface (Table 1), in agreement with data reported in the literature (39). The EE was rather low, in comparison to other vesicular formulations (3940) and was not influenced by the different molecular properties (Table 1) and, for this reason, release studies were carried out on non purified samples. Only the entrapment of NR was relevant, due to its high lipophilicity.

$\begin{aligned} & \text { Table 1. Vesicle dimensions (diameter), polydispersity } \\
& \text { (\%).Reported data are means of three experiments } \pm \text { S.D. }\end{aligned}$
\begin{tabular}{ccccc} 
Somple & Size $(\mathrm{nm})$ & p.i. & $\zeta$-potential values & and vesicle EE \\
\hline Empty-NSV & $138 \pm 1$ & $0.051 \pm 0.03$ & $-39 \pm 1$ & EE (\%) \\
NSV-NR & $140 \pm 1$ & $0.252 \pm 0.06$ & $-40 \pm 3$ & ---- \\
NSV-IBU 1\% & $150 \pm 2$ & $0.130 \pm 0.01$ & $-44 \pm 2$ & $73.0 \pm 2.0$ \\
NSV-CAFF 3\% & $168 \pm 1$ & $0.147 \pm 0.02$ & $-39 \pm 1$ & $0.4 \pm 0.1$ \\
NSV-CALC & $180 \pm 7$ & $0.183 \pm 0.11$ & $-44 \pm 1$ & $0.2 \pm 0.1$ \\
\end{tabular}



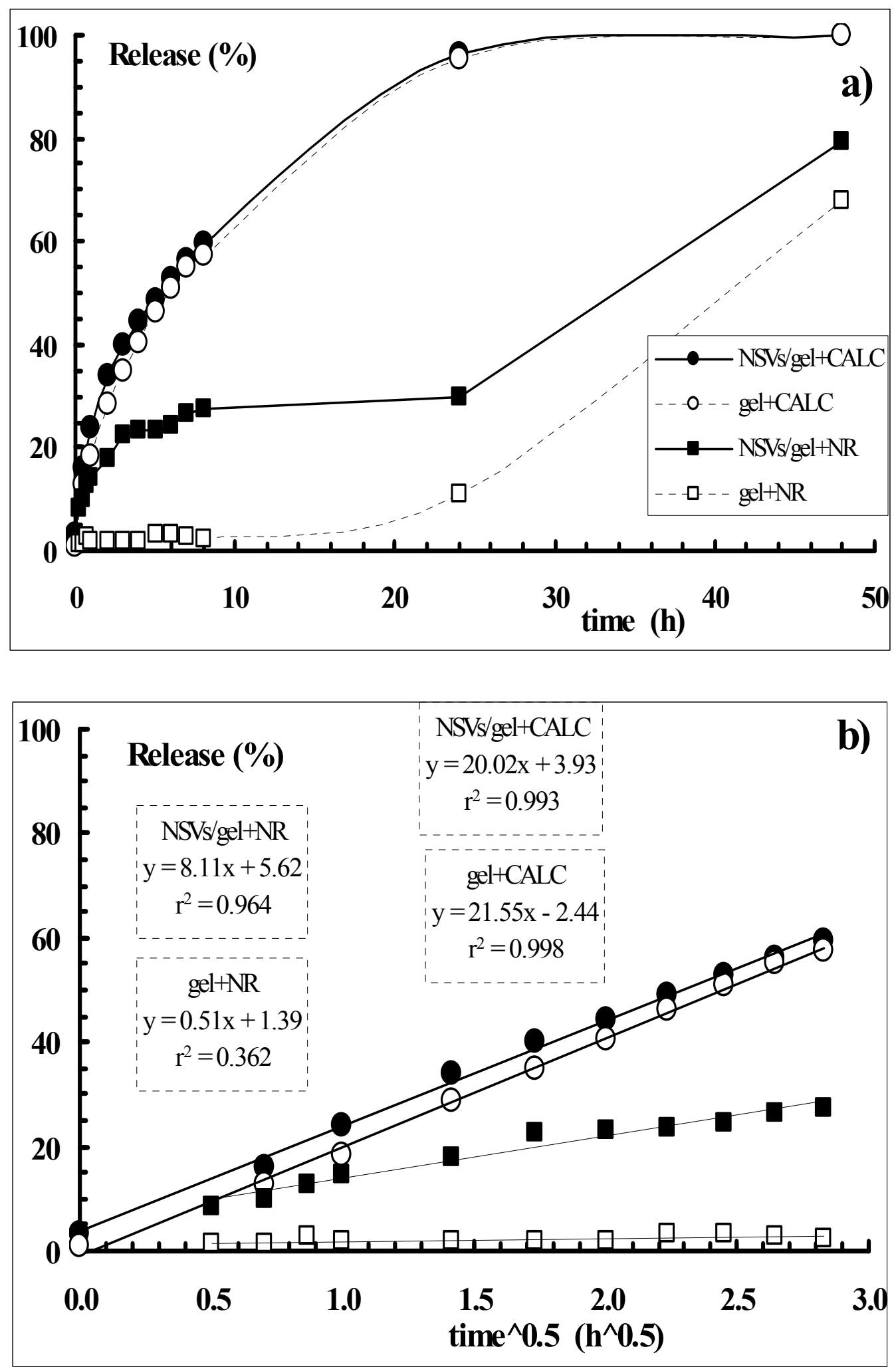

Figure 2. Release profiles of CALC and NR from the LBG/Xanth gel (empty symbols) and from the loaded NSVsLBG/Xanth systems (full symbols) in HEPES ( $\mathrm{pH}=7.4$ ) and $32^{\circ} \mathrm{C}$. (a) As a function of time; (b) as a function of the square root of time. Release experiments were carried out in triplicate. The reported value represents mean values and lay within $10 \%$ of the mean. 

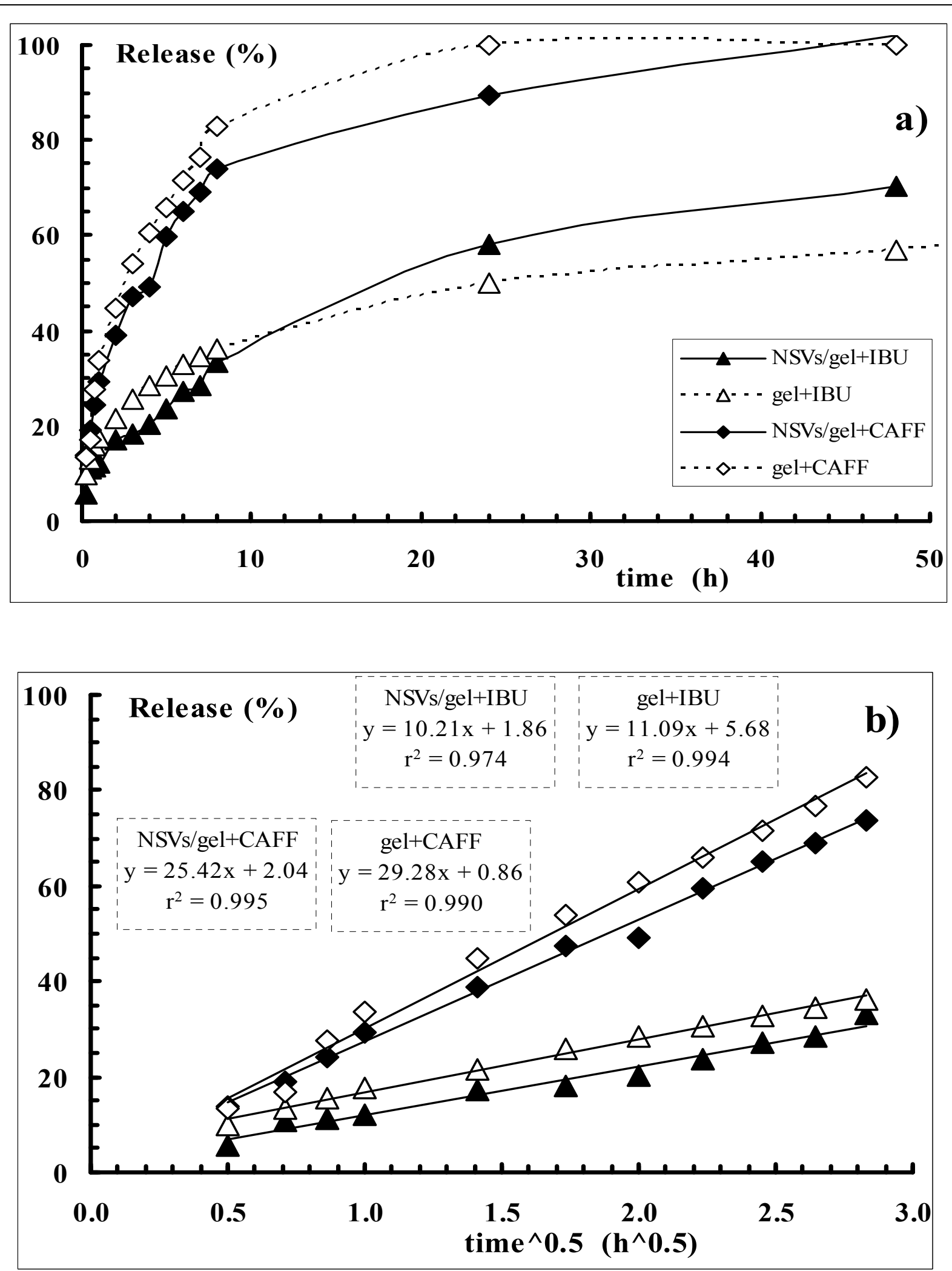

Figure 3. Release profiles of IBU and CAFF from the LBG/Xanth gel (empty symbols) and from the loaded NSVsLBG/Xanth systems (full symbols) in HEPES ( $\mathrm{pH}=7.4)$ and $32^{\circ} \mathrm{C}$. (a) As a function of time; (b) as a function of the square root of time. The release experiments were carried out in triplicate. Reported values represent mean values and lay within $10 \%$ of the mean. 


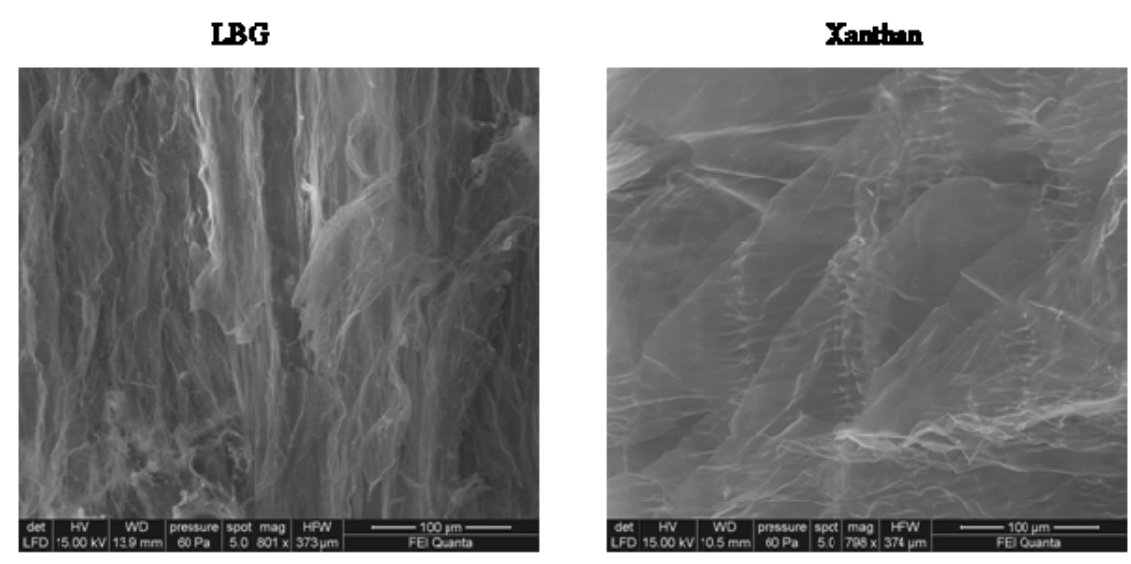

LBG-Xan'h 1:1

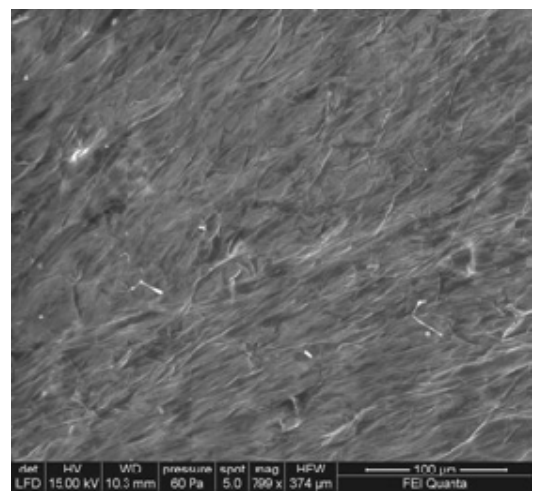

Figure 4. Scanning electron micrograph of surface morphology of freeze-dried samples of LBG, Xanth (top), and LBG/Xanth 1:1 (bottom) prepared at $60^{\circ} \mathrm{C}(800 \mathrm{x})$.

It has been previously reported (44) that the Tw20-IBU vesicular system does not induce an appreciable variation of IBU cutaneous permeability but allows an IBU depot in the skin. Taking into account such evidence and that aim of this study was the evaluation of the hydrogel effect on the structural integrity of vesicles during the release process, IBU was chosen as model drug for this in vitro evaluation.

Actually, to our best of knowledge, the present work represents one of the few reports on the potential application of a combined system vesicles/hydrogel in drug delivery (45-48).

Netherless it must be pointed out that while previous papers were mainly related to the release of selected drugs from the overall vesicle/hydrogel combined system, in the present case our attention was focused on the possibility of finding a hydrogel system capable to release intact drug-loaded vesicles.

The release from the NSVs-gel systems of two probes, the hydrophilic CALC and the hydrophobic NR, is shown in Figure 2a, together with the release from the polysaccharidic gel matrix, i.e. without NSVs.
The comparison between the two curves indicates that the diffusion of CALC through the gel was not affected by the entrapment in the NSVs (100\% release in 24 hours). In fact, the NSVs were capable of diffusing without being broken or damaged by the polymeric matrix in which they are embedded (Table 2).

On the other hand, the results showed that the presence of vesicles loaded with NR was crucial. When NR was directly dispersed in the hydrogel, almost no diffusion occurred due to its high hydrophobicity that inhibits the release of the molecule in the diffusion medium while, when the probe was previously loaded inside the vesicles, an appreciable release was monitored and almost $70 \%$ of NR was delivered within $48 \mathrm{~h}$ (Figure 2a).

Turbidity, dimension and zeta potential analyses carried out on the diffusion medium (Table 2) indicate that the loaded niosomes are released by the hydrogel matrix without being damaged. Furthermore, the release of the two probes obeys a Fickian diffusion (Figure 2b). 


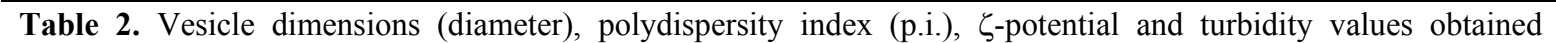
analyzing the surrounding medium at the end of the release experiments. Reported data are mean of three experiments \pm S.D.

\begin{tabular}{cccccc}
\hline Sample & Size $(\mathrm{nm})$ & p.i. & S-potential $(\mathrm{mV})$ & $\begin{array}{c}\text { Turbidity } \mathrm{t}=0 \\
(\mathrm{AU})\end{array}$ & $\begin{array}{c}\text { Turbidity } \mathrm{t}=48 \mathrm{~h} \\
(\mathrm{AU})\end{array}$ \\
\hline NSV-CALC & $223 \pm 4$ & $0.334 \pm 0.08$ & $-25 \pm 1$ & 42 & 205 \\
NSV-NR & $191 \pm 2$ & $0.369 \pm 0.05$ & $-27 \pm 3$ & 75 & 150 \\
NSV-IBU 1\% & $206 \pm 5$ & $0.354 \pm 0.07$ & $-23 \pm 2$ & 36 & 220 \\
NSV-CAFF 3\% & $202 \pm 3$ & $0.328 \pm 0.06$ & $-21 \pm 1$ & 53 & 198 \\
\hline
\end{tabular}

The release profiles of two model drugs, CAFF and IBU, obtained from the NSVs-gel systems and from the hydrogel without vesicles (Figure 3) showed an appreciable difference between the delivery of the two drugs. The more hydrophilic CAFF (solubility in water, $21.7 \mathrm{mg} / \mathrm{mL}, 25^{\circ} \mathrm{C}$ ) was released much faster than IBU and it was capable to diffuse out completely from the polymeric matrix within the first 24-48 hours, with a slight lower delivery rate in the presence of NSVs. The comparison between the two curves indicates that the diffusion of the drug through the network was not much affected by the preliminary entrapment in the NSVs. The release appeared to be only slightly faster in the absence of NSVs with the delivery profiles almost superimposable during the first 8 hours.

A rather different behaviour was detected when IBU, i.e. a hydrophobic drug (very slightly soluble in water, $<1 \mathrm{mg} / \mathrm{mL}, 25^{\circ} \mathrm{C}$ ), was tested. The results showed that, even after $48 \mathrm{~h}$, the release was not complete and only about $60 \%$ of the loaded drug was capable to diffuse from the matrix (Figure 3a). Also in this case there was only a slight difference between the two release profiles during the first 8 hours, indicating that the presence of the vesicles did not appreciably influence the diffusion of the guest molecules. The significant delivery of the hydrophobic IBU, also in the absence of NSVs, can be related to the increase of solubility of this drug at $\mathrm{pH}=7.4$ (IBU $\mathrm{pKa}=4.3$ ), where it was present also in the dissociated form. Of course, such effect cannot be taken into account in the case of NR. Also in the case of model drugs, turbidity, dimension and zeta potential analyses (Table 2) indicated that the loaded niosomes were released by the hydrogel matrix without being damaged. Moreover, data reported in Table 2, showed that niosomes released by the hydrogel matrix were characterized by an increase of dimensions and a decrease of the absolute zeta potential values; that can be related to a possible interaction between vesicle surfaces and drug molecules, not evidenced during the release of model probes.

Furthermore, comparing the release profiles of the two hydrophobic molecules (Figures 2a and 3a) it was possible to observe that in both cases the diffusion follows a Fickian mechanism (Figure $3 \mathrm{~b})$ and that, regardless of the degree of ionization, the maximum release was about $60 \%$ during the first $48 \mathrm{~h}$. This evidence may be related to the role of the vesicular system that was able to diffuse intact through the hydrogel matrix, while simultaneously driving, the hydrophobic molecules into the external medium. From this effect, it was evident that the hydrophilic molecules (100\% released in 24 hours, Figures 2a and $3 \mathrm{a}$ ) are capable of diffusing by themselves, also in the absence of vesicular carriers.

SEM micrographs (Figure 4), taken on freezedried samples, supported the above discussed considerations: it was clear how the texture of the LBG/xanth mixture was much more compact in comparison to that of the single polymers. Thus, the formation of this supramolecular structure led to a slow release of the entrapped vesicles. As already reported (49), when the two polymer solutions were mixed at a high temperature ( $\mathrm{T}>\mathrm{T}_{\text {melting }}$ of xanth chains), a self-sustaining gel was obtained and the strength of the obtained network corresponded to storage moduli of the order of hundreds of $\mathrm{Pa}$, while the two single polymer solutions had negligible values.

\section{CONCLUSIONS}

The new formulation, based on loaded vesicles entrapped inside a polymeric network, appears to be suitable for topical application. In fact, the combination of the two carriers, the niosomes and the LBG/xanth gel, leads to a real improvement in the slow release of drugs for dermal diseases. Once the intact vesicles are delivered outside of the hydrogel matrix they can act as carriers for enhanced dermal delivery. Thus, reported in vitro experiments, show the capability of the novel 
formulation to combine the qualities of both chosen single systems: the polymeric hydrogel slows down the delivery of the niosomes and the niosomes themselves, once diffused out of the matrix, are capable to act as carriers for the loaded molecules. Moreover, the vesicular structures, at the same time, may enhance the delivery of the guest molecules, both hydrophilic and hydrophobic ones, in the stratum corneum due to their specific constituents.

\section{ACKNOWLEDGEMENTS}

Financial supports from University "Sapienza", Faculty Funding (2009), and from FIRB, Fondo per gli Investimenti della Ricerca di Base, Research Program: Ricerca e Sviluppo del Farmaco (CHEM-PROFARMA-NET), grant no. RBPR05NWWC_003 are acknowledged.

\section{REFERENCES}

1. Muller RH, Radtke M, Wissing SA. Solid lipid nanoparticles (SLN) and nanostructured lipid carriers (NLC) in cosmetic and dermatological preparations. Adv Drug Deliv Rev. 2002; 54: S131-S155.

2. Uchegbu IF, Vyas SP. Non-ionic surfactant vesicles (Niosomes) in drug delivery. Int $\mathrm{J}$ Pharm 1998; 17: 33-70.

3. Santucci E, Carafa M, Coviello T, Murtas E, Riccieri FM, Alhaique, F, Modesti A, Modica A.. Vesicles from polysorbate 20 and cholesterol. A simple preparation and characterization. STP Pharma Sci. 1996; 6 : 29-32.

4. Di Marzio L, Marianecci C, Petrone M, Rinaldi F, Carafa M Novel $\mathrm{pH}$-sensitive non-ionic surfactant vesicles: comparison between Tween 21 and Tween 20 Colloids and Surface B: Biointerfaces 2011; 82: 18-24.

5. Masotti A, Vicennati P, Alisi A, Marianecci C, Rinaldi F, Carafa M, Ortaggi G Novel Tween ${ }^{\circledR}$ 20 derivatives enable the formation of efficient $\mathrm{pH}$-sensitive drug delivery vehicles for human hepatoblastoma. Bioorganic \& Medicinal Chemistry Letters 2010; 2: 3021-3025.

6. Maibach HI, Choi MJ. Liposomes and niosomes as topical drug delivery systems. Skin Pharmacol Physiol 2005; 18: 209-219.

7. L'Oréal, (1975) Procédé de fabrication de dispersions aqueuses de spérules lipidiques et nouvelles compositions correspondantes French Patent 2315991

8. L'Oréal, (1989) Cosmetic and pharmaceutical compositions containing niosomes and a watersoluble polyamide, and a process for preparing these compositions, US Patent 4830857.

9. Carafa M, Marianecci C, Lucania G, Marchei E, Santucci E. New vesicular ampicillin-loaded delivery systems for topical application: characterization, in vitro permeation experiments and antimicrobial activity. J Contr. Rel. 2004; 95:67-74.

10. Scott DC, Clymer JW Estimation of Distribution Coefficients from the Partition Coefficient and pKa. www.pharmtech.com 2002

11. Kaliszan R, Haber P, Tomasz B, Siluk D, Valko K Lipophilicity and $\mathrm{pKa}$ estimates from gradient high-performance liquid chromatography J. Chrom. A, 2002; 965: 117-127

12. Coviello T, Matricard P, Marianecci C, Alhaique F Polysaccharide hydrogels for modified release formulations. J. Control. Release, 2007; 119: 5-24.

13. Coviello T, Matricardi P, Alhaique F Drug delivery strategies using polysaccharidic gels. Expert Opin. Drug Deliv. 2006; 3: 395-404.

14. Morris, E.R., Mixed polymer gels. In P. Harris (Ed.). Food Gels. Elsevier London, UK, pp 291359, 1990.

15. Coviello T, Kajiwara K, Burchard W, Dentini M, Crescenzi V. Solution properties of Xanthan. 1. Dynamic and static light scattering from native and modified Xanthans in dilute solutions. Macromolecules, 1986; 19: 2826-2831.

16. Crescenzi V, Dentini M, Coviello T Solution and gelling properties of polysaccharide polyelectrolytes Biophys. Chem. 1991; 41: 61-71.

17. Lundin L, Hermansson A. M. Supermolecular aspects of Xanthan-locust bean gum gels based on rheology and electron microscopy. Carbohydrate Polymers 1995; 26: 129-140.

18. Richter S, Boyko V, Matzker R, Schröter K. Gelation studies: comparison of the critical exponents obtained by dynamic light scattering and rheology, $2^{\mathrm{a}} \mathrm{A}$ thermoreversible gelling system: mixtures of xanthan gum locust bean gum. Macromolecular Rapid Communication 2004; 25 : 1504-1509.

19. Richter S, Brand T, Berger S. Comparative monitoring of the gelation process of a Thermoreversible gelling system made of xanthan gum and locust bean gum by dynamic light scattering and ${ }^{1} \mathrm{H}$ NMR spectroscopy. Macromolecular Rapid Communication 2005; 26 : 548-553.

20. Wang E, Wang Y J, Sun Z. Conformational role of xanthan in its interaction with locust bean gum. Journal of Food Science 2002; 67: 2609-2614.

21. Zhan DF, Ridout MJ, Brownsey G J, Morris V J Xanthan-locust bean gum interactions and gelation. Carbohydrate Polymers 1993; 21: 53-58.

22. Dolz M, Her Delegido J, Alfaro M C, Muňoz J. Influence of xanthan gum and locust bean gum upon flow and thixotropic behaviour of food emulsions containing modified starch. Journal of Food Engineering, 2007; 81: 179-186.

23. Mandala I, Kapetanakou A, Kostaropoulos A. Physical properties of breads containing hydrocolloids stored at low temperature: II-Effect 
of freezing. Food Hydrocolloids 2008; 22: 14431451.

24. Pedersen J K Carrageenan, pectin and xanthan/locust bean gum gels. Trends in their food use. Food Chemistry 1980; 6: 77-88.

25. Sahin H, Ozdemir F. Effect of some hydrocolloids on the rheological properties of different formulated ketchups. Food Hydrocolloids 2004; 18: 1015-1022.

26. Ramírez J A, Barrera M, Morales OG, Vázquez M. Efefct of xanthan and locust bean gums on the gelling properties of myofibrillar protein. Food Hydrocolloids 2002 ; 16: 11-16.

27. Secouard S, Malhiac C, Grisel M, Decroix B. Release of limonene from polysaccharide matrices: viscosity and synergy effect. Food Chemistry 2003; 82: 2227-234.

28. Baichwal, AR (2004). Sustained-release matrix for high-dose insoluble drugs. US Patent Office, Pat. No. 2004121012.

29. Fadden DJ, Kulkarni NM, Sorg AF (2004). Fast dissolving orally consumable films containing a modified starch for improved heat moisture resistance. US Patent Office, Pat. No. 2004247648.

30. Mannion RO., Melia C D., Mitchell JR., Harding SE., Green AP. Effect of xanthan/locust bean gum synergy on ibuprofen release from hydrophilic matrix tablets. Journal of Pharmacy and Pharmacology 1991; S 43: 79P-79P.

31. Rolf D (2003). Aqueous gel wound dressing and package. US Patent Office, Pat. No. 6620436.

32. Sugden K. (1989). Buccal etorphine tablets with xanthan and locust bean gums. US Patent Office, Pat. No. 4829056.

33. Ughini F, Andreazza IF, Ganter JLMS, Bresolin TMB. Evaluation of Xanthan and highly substituted galactomannan from M. scabrella as a sustained release matrix. Int. J. Pharm. 2004; 271: 197-205.

34. Venkataraju MP, Gowda DV, Rajesh KS, Shivakumar HG. Xanthan and locust bean gum (from Ceratonia siliqua) matrix tablets for oral controlled delivery of metoprolol tartrate. Current Drug Therapy 2008; 3: 70-77.

35. Coviello T, Alhaique F, Dorigo A, Matricardi P, Grassi M. Two galactomannans and scleroglucan as matrices for drug delivery: preparation and release studies Eur. J. Pharm. Biopharm. 2007; 66: 200-209.

36. Sandolo C, Bulone D, Mangione M, Margheritelli S, Di Meo C, Alhaique F, Matricardi P, Coviello T. Synergistic interaction of Locust Bean Gum and Xanthan investigated by rheology and light scattering Carboydr. Polym. 2010; 82: 733-741.

37. Kok M S, Hill SE, Mitchell J R. Viscosity of galactomannans during high temperature processing: influence of degradation and solubilisation. Food Hydrocolloids 1999;13: 535542.

38. Centis V, Vermette P. Physico-chemical properties and cytotoxicity assessment of PEG-modified liposomes containing human hemoglobin. Colloids Surf B Biointerfaces 2008; 65:239-246

39. Mohammed AR, Weston N, Coombes AGA, Fitzgerald M, Perrie Y. Liposome formulation of poorly water-soluble drugs: optimisation of drug loading and ESEM analysis of stability. Int $\mathrm{J}$ Pharm 2004; 285:23-34.

40. Khazaeli P, Pardakhty A, Shoorabi H. Caffeineloaded niosomes: characterization and in vitro release studies Drug Delivery 2007; 14: 447-452 .

41. Saettone MF, Perini G, Carafa M, Santucci E, Alhaique F. Non-ionic surfactant vesicles as ophthalmic carriers for cyclopentolate. A preliminary evaluation. S. T. P. Pharma.Sci. 1996; 6:94-98

42. Terzano C, Allegra L, Alhaique F, Marianecci C, Carafa M. Non phospholipid vesicles for pulmonary glucocorticoid delivery Eur. J. Pharm. Biopharm. 2005; 59: 57-62.

43. Marianecci C, Paolino D, Celia C, Fresta M, Carafa M, Alhaique F. Non-ionic Surfactant Vesicles, Beclomethasone Dipropionate, Pulmonary Drug Carrier, Primary Human Lung Fibroblasts, Carrier-cell interaction, Antiinflammatory activity Journal of Controlled Release 2010; 147: 127-135.

44. Carafa M, Marianecci C, Rinaldi F, Santucci E, Tampucci S, Monti D Span and Tween neutral and $\mathrm{pH}$-sensitive vesicles: characterization and in vitro skin permeation. J Liposome Res. 2009; 19:33240.

45. Hosny KM Optimization of gatifloxacin liposomal hydrogel for enhanced transcorneal permeation J. Lip. Res. 2010; 20: 31-37

46. Kapadia R, Khambete H, Katara R, Ramteke S A Novel Approach For Ocular Delivery of Acyclovir Via Niosomes Entrapped In Situ Hydrogel System J. Pharm. Res. 2009; 2: 745-751

47. Fang JY, Hsu SH, Leu YL, Hu JW Delivery of cisplatin from pluronic co-polymer systems: Liposome inclusion and alginate coupling $\mathrm{J}$. Biomat. Sci., Polymer Edition 2009; 20: 10311047

48. Alcantar N, Williams EC, Toomey R. NiosomeHydrogel Drug Delivery System Patent US 20100682642010 )

49. Sandolo C, Coviello T, Matricardi P, Alhaique F. Characterization of polysaccharide hydrogels for modified drug delivery Eur. Biophys. J.2007; 36: 693-700 